\title{
Antiphospholipid Antibodies In Adults With Ischaemic Stroke Seen At The University of Benin Teaching Hospital, Benin City, Nigeria
}

\author{
Nwogoh Benedict, Awodu Omolade Augustina, Ogunrin A. Olubunmi, \\ Nwannadi Ikenna Alexander
}

\section{Introduction}

Antiphospholipid antibodies (APLA) are auto-antibodies that recognize various combinations of phospholipids, phospholipid-binding proteins or both and thus prolong the phospholipid dependent coagulation tests in vitro., 2 There are different subgroups of APLA. The most characterize include lupus anticoagulant (LA), anticardiolipin (ACA), anti- glycoprotein 1 based on detection methods. ${ }^{1}$

Antiphospholipid antibodies (APLA) are associated with a protean of clinical manifestations affecting virtually every organ of the body. They have been associated with recurrent episodes of arterial or venous thrombosis or both, recurrent fetal loss, neurological diseases and possible thrombocytopenia. ${ }^{3,4}$

Stroke is defined as a syndrome of rapidly developing clinical symptoms and signs of focal (or global) loss of cerebral functions, with symptoms lasting 24 hours or longer or resulting in death with no apparent cause other than of vascular origin 5 .

The prevalence of stroke in Nigeria is on the increase.6, 7 Osuntokun et al6 reported a crude prevalence of 58 per 100,000 of the population and Danesi et $a l^{7}$ in a more recent studies reported a rate of 1.14 per 1,000 . It is principally a disease of the elderly ${ }^{8}$ however there is an increase in the prevalence of stroke in younger age group $^{9,10}$. Stroke is a devastating event because of the consequent physical and psychological stress, economic loss to families and to the nation especially when young people are affected.

Several studies ${ }^{11-13}$ have demonstrated a significant association of APLA and stroke while some others ${ }^{14,15}$ did not. Thus role of APLA as an independent risk factor for stroke is controversial. Most of these studies were conducted in none Africans. There is a need for local studies to determine the role of APLA in stroke in our environment.

\section{OBJECTIVES}

The objectives of this study are to determine the prevalence of APLA in adults with Ischaemic Stroke, to determine the risk of developing stroke in APLA positive Ischaemic stroke patients and to determine if there is any correlation between APLA and haematological parameters.

\section{Materials And Methods}

This is a case control study conducted at the University of Benin Teaching Hospital (UBTH). The centre is a tertiary health facility located in Benin City, Edo state, Nigeria. The hospital serve as a research centre, a training institute for undergraduate and postgraduate medical (including residency) training as well as a major referal centre serving Edo and neighbouring Delta, Kogi and Ondo states.

A total of 90 subjects were recruited for the study. The case group comprises of 60 ischaemic stroke patients aged 18 - 60 years with a CT scan evidence of ischaemic stroke and the controls consist of 30 Non stroke patients presenting to the medical outpatient for a routine clinical check. All participants gave consent to participate in the study. The study was approved by the hospital ethical committee.

Patients on anticoagulant therapy, those with coagulation factor deficiencies, infectious diseases (including HIV), malignancies and those on steroid therapy were excluded.

About $12 \mathrm{mls}$ of blood was collected from the antecubital vein of each of the study subject. $4.5 \mathrm{mls}$ was dispensed into a sample bottle containing $0.5 \mathrm{mls}$ of $3.2 \%(0.109 \mathrm{M})$ trisodium citrate anticoagulant. The citrated samples were centrifuged at $1500 \mathrm{rpm}$ for 15 minutes and the supernatant (platelet poor plasma) was separated into a plain tube and stored at $-80^{\circ} \mathrm{C}$ in the blood bank freezer. The citrated sample was used for LA assay. Another $5 \mathrm{mls}$ of blood was dispensed into a plain container, allowed to clot and retract. The clotted saample was centrifuged at $1500 \mathrm{rpm}$ for $10 \mathrm{mins}$ and the supernatant (serum) was separated into another plain container and stored in a $-800 \mathrm{C}$ freezer until analysed.

The remaining $2.5 \mathrm{mls}$ of blood was dispensed into an ethylenediaminetetrachloroacetic acid (EDTA) container. The sample was analysed for full blood count immediately. 


\section{Antiphospholipid Assay:}

Lupus Anticoagulant Assay: Dilute Russel Viper venom test (DRVVT) was used to screen for LA. Mixing test was done on samples with prolonged screening results and DRVVT confirm test was used for confirmation. LA screen kits (LOT 6Y12B00) and LA confirm kits (LOT 6X12B00) by TECHNOCLONE DIAGNOSTICS GmbH, Vienna were used for the analysis

Anticardiolipin Assay: ACA titer was measured using an enzyme immunoassay test kit by Bioquant Diagnostics, San Diego, USA.

Full Blood Count: Blood count was estimated using Haematology automated analyzer MODEL SYSMEX KN21, Japan.

\section{Data Analysis}

Data were analyzed using the SPSS 16. LA and ACA results of test and control were compared using Chi-square test or Fishers Exact test as appropriate. Student's T test was used to compare means of numerical variables. The risk of stroke associated with APLA was tested using odd ratios (OR). The relationship between blood counts and APLA was estimated using Spearman's correlation test. $P<0.05$ was regarded as significant.

\section{Results}

The mean age for the ischaemic stroke patients and controls were $48.5 \pm 9.4$ years and $42.9 \pm 9.5$ years respectively. The stroke subjects comprise 36 males and 24 females while the controls included 16 males and 14 females as in Table 1. Forty two (72.4\%) of the stroke patients are on antiplatelet (low dose aspirin).

Six $(10 \%)$ of subjects with ischaemic stroke were positive for LA while none of the controls had LA. Twelve $(20 \%)$ of the stroke subjects were positive for ACA while two $(6.7 \%)$ of the controls were positive (table 1). Only one of the subjects was positive for both APLAs. The prevalence of APLA in ischaemic stroke patients was $28.3 \%$ as against $6.7 \%$ in the controls. This was statistically significant with a $P=0.026$ and an odd ratio of 5.53 .

There was no sex preponderance in APLA positivity in the ischaemic stroke group. The identifiable risk factors for stroke in the APLA positive and APLA negative case group are presented in table 2. Alcohol intake and absence of any identifiable risk factor were noted to be significantly higher in the APLA positive stroke patients. (Table 2)

There was no significant difference in the platelet counts between the case and control groups as in table 3. There was no correlation between platelets and APLA antibodies (table 4).

\section{Discussion}

Antiphospholipid antibodies (APLA) are acquired procoagulant antibodies which bind negatively charged phospholipid moieties and are associated with an increased risk of thrombosis including ischaemic stroke. In this study, the prevalence of APLA was found to be $28.3 \%$. There are limited studies on antiphospholipid antibodies in stroke patients in our environment. Several studies have reported an increase in the prevalence of APLA in ischaemic stroke patients but there are inconsistencies in their association as a risk factor for stroke.

A number of studies on APLA were based either on LA or ACA assay alone. The prevalence of LA in ischaemic stroke as reported in various studies ranged from $4-20.9 \%{ }^{16,17}$ while that of ACA ranged from 6 $38 \%{ }^{15,18}$ depending on selection criteria of the subjects and the method for detection of APLA.

Montalba et $a l^{19}$ studied 389 patients with cerebral ischemia and found $16(4.1 \%)$ to have the lupus anticoagulant. Similarly, Trimble et al in a prospective study on 51 patients with stroke reported a prevalence of $6 \%$. However, Muir et al reported a high prevalence of ACA of $38 \%{ }^{15}$ in a case control study of 226 ischaemic

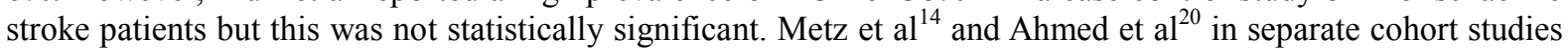
reported rates of $12 \%$ and $11.4 \%$ respectively. The study by Metz et al was based on the use of three different detection assays.

The prevalence in this study is within the range reported in these studies. Most of these studies reported a consistently increased prevalence of APLA in stroke, while some ${ }^{11-13}$ found APLA to be a risk factor for stroke, some others ${ }^{14,15}$ did not. In this study, APLA is increased in the cases group and this was statistically significant and thus a risk factor for stroke in our study population. The odd for detecting APLA in ischaemic stroke patients is 5.53 times higher than in control.

Nencini et $\mathrm{al}^{12}$ and some other researchers have reported a female preponderance of APLA. In this study, though the proportion of females positive for APLA was higher than that of males with an OR of 1.57, this was not statistically significant. The female preponderance reported in some of these studies may have been due some bias introduced by the female preponderance of autoimmune diseases such as systemic lupus erythematosis in the studied subjects. Studies which excluded subjects with autoimmune diseases have failed to show such female preponderance. 
This study reported a high prevalence of multiple risk factors for stroke such as hypertension, cardiac diseases, hyperlipidaemia, diabetes mellitus, smoking and alcoholism. The presence of multiple cerebrovascular risk factors may have contributed to the high prevalence of APLA in this study. Some studies have reported a high prevalence of APLA in subjects with undetermined risk factors for ischaemic stroke, ${ }^{20}$ similarly we found APLA to be significantly present in stroke subjects with undetermined risk factor. This is at variance with the findings Janardhan et $\mathrm{al}^{11}$ and Tanne et $\mathrm{al}^{21}$ in their different studies. Blohorn et $\mathrm{al}^{22}$ however reported a significantly higher prevalence of hyperlipidaemia and alcohol abuse in APLA positive ischaemic stroke patients. Thus the presence of multiple risk factors may be contributory to the positive APLA in these studies.

The mechanisms by which APLA could lead to stroke and other thrombotic manifestations are multiple. Effects on platelets, coagulation proteins, and endothelial cells by APLA have been demonstrated and support the plausibility of APLA associated thrombosis. For example, APLA from patients with thrombosis, thrombocytopenia, or fetal loss can induce a dose-dependent increase in the activation and aggregation of human platelets. ${ }^{23}$ This effect appears to be mediated through binding to phosphatidylserine the most common phospholipid in cell membranes, or -glycoprotein 1, a glycoprotein APLA cofactor. This effect appears to be mediated through binding to phosphatidylserine the most common phospholipid in cell membranes, or glycoprotein I, a glycoprotein APLA cofactor. This study did not show any correlation between APLA and platelet count. This could be because in most of the subjects, the event occurred several months ago prior to the sampling for this study. Furthermore, majority of the subjects are on antiplatelet therapy as a form of secondary prophylaxis. This may reduce the rate of platelet activation and consumption in the studied subjects.

\section{Conclusion}

This study has found the prevalence of APLA in ischaemic stroke patients of $28.30 \%$ and has demonstrated that APLA is a risk factor for stroke. There is no sex preponderance in the prevalence of APLA in ischaemic stroke patients. There are multiple risk factors for stroke in APLA positive subjects. APLA assay may be recommended for patients at risk of ischaemic stroke.

\section{References}

[1]. Ahmed E, Stegmayr B, Trifunovic J et al. Anticardiolipin antibodies are not an independent risk factor for stroke. Stroke 2000; 31: $1289-1293$.

[2]. Asherson RA, Harris EN. Anticardiolipin Antibodies: Clinical Associations. Postgrad Med J. 1986; 62:1081-087.

[3]. Blohorn A, Guegan-Massardier E, Triquenot A et al. antiphospholipid antibodies in the acute phase of cerebral ischaemia in young adults: A Descriptive study on 139 patients. Cerebrovasc Dis 2002; 13: 156 - 162.

[4]. Brey RL, Stallworth CL, McGlasson DL et al. Antiphospholipid antibodies and stroke in young women. Stroke 2002:33; 2396 2401.

[5]. Danesi M, Okubadejo N, Ojini F. Prevalence of Stroke in an Urban, Mixed-Income Community in Lagos, Nigeria. Neuroepidemiology 2007; 28 (4): 216 - 223.

[6]. de Groot PG, Derksen RHWM. Specificity and clinical relevance of lupus anticoagulant. Vessels 1995; 1:22 - 26

[7]. Desalu OO, Wahab KW, Fawale B et al. A review of stroke admissions at a tertiary hospital in rural Southwestern Nigeria. Ann Afr Med 20011; 10: 80-85.

[8]. Hart RG, Miller VT, Coull BM et al. Cerebral infarction associated with lupus anticoagulants: Preliminary report. Stroke 1984;15:114-118

[9]. Hughes GRV. Thrombosis, Abortion Cerebral Disease and Lupus Anticoagulant. BMJ 1983; 287:1088-1089.

[10]. Janardhan V, Wolf PA, Kase CS et al. Anticardiolipin antibodies and risk of ischaemic stroke and transient ischaemic attack. Stroke $2004 ; 35: 736-741$.

[11]. Levine JS, Branch W, Rauch J. The Antiphospholipid Syndrome. N Engl J Med 2002; 346: 752-763

[12]. Lutters BC, Derksen RH, Tekelenburg WL et al. Dimers of beta 2-glycoprotein I increase platelet deposition to collagen via interaction with phospholipids and the apolipoprotein E receptor 2'. J Biol Chem 2003; 278:33831-33838.

[13]. Metz LM, Edworthy S, Mydlarski R et al. The frequency of phospholipid antibodies in an unselected stroke population. Can J Neurol Sci. 1998; 25: $64-69$.

[14]. Montalban J, Codina A, Ordi J et al. Antiphospholipid antibodies in Cerebral Ischaemia. Stroke 1991; 22(6): 750 - 753

[15]. Muir KW, Squire IB, Lees KR, Alwan W. Anticardiolipin antibodies in an unselected stroke population. Lancet 1994; 344 (8920): $452-456$.

[16]. Nencini P, Baruffi MC, Abbate R et al. Lupus anticoagulant and anticardiolipin antibodies in young adults with cerebral ischaemia. Stroke 1992; 23: 189 - 193.

[17]. Njoku CH, Aduloju AB. Stroke in Sokoto, Nigeria: A five year retrospective study. Ann Afr Med. $2004 ; 3$ (2): 73 - 76.

[18]. Onwuchekwa ACA, Onwuchekwa RCA, Asekomeh EGA. Stroke in young Nigerian adults. J Vasc Nursing 2009 ; $27(4)$ : 98 - 102.

[19]. Osuntokun BO, Bademosi O, Akinkugbe OO et al. Incidence of Stroke in an African City: Results from the stroke registry at Ibadan, Nigeria, 1973-1775. Stroke 1979; 10: 205-207.

[20]. Piero V, Levine SR, Tietjen GE. Cerebrovascular ischaemic events with high positive anticardiolipin antibodies. Stroke 1998; 29:2245-2253.

[21]. Rand JH, Levin MB, Alving BM. The Antiphospholipid Syndrome: Clinical Presentation, Diagnosis and Patient Management. In: Kitchens CS, Alving BM, Kessler CM, eds. Consultative Hemostasis and Thrombosis. 2nd ed. Philadelphia, PA; WB Saunders. 2007: 269-278.

[22]. Tanne D, Olhaberriague LD, Schultz LR et al. Anticardiolipin antibodies and their associations with cerebrovascular risk factors. Neurology 1999; 52 (7): 1368.

[23]. Wolf PA, Kannel WB, Dawber TR. Prospective investigations: The Framingham study and the epidemiology of stroke. Advances in Neurology 1978; 19: 107-120. 
Table 1:

\begin{tabular}{llll}
\hline Variables & $\begin{array}{l}\text { Ischaemic Stroke } \\
(\mathbf{N = 6 0 )}\end{array}$ & $\begin{array}{l}\text { Control } \\
(\mathbf{N = 3 0 )}\end{array}$ & P-values \\
\hline Age (Mean + SD & $48.5 \pm 9.4$ & $42.9 \pm 9.5$ & $>0.05$ \\
Sex & & & \\
Males & 36 & 16 & $>0.05$ \\
Females & 24 & 14 & 0.026 \\
M:F & $1.5: 1$ & $1.14: 1$ & 0.173 \\
APLA & $17(28.3 \%)$ & $2(6.7 \%)$ & 0.230 \\
LA positive & $6(10.0 \%)$ & $0(0.0 \%)$ & 5.53 \\
ACA positive & $12(20.0 \%)$ & $2(6.7 \%)$ & \\
LA/ACA positive & $1(1.7 \%)$ & $0(0.0 \%)$ & \\
\hline
\end{tabular}

Table 2:

\begin{tabular}{llll}
\hline Variables & APLA Positive & APLA Negative & P-value \\
\hline Age & $50.2 \pm 5.4$ & $47.9 \pm 10.5$ & 0.393 \\
Sex & $(\%)$ & & $27(62.8)$ \\
$\quad$ Males & $9(52.9)$ & $16(37.2)$ & 0.564 \\
Females & $8(47.1)$ & $(\%)$ & 0.932 \\
Risk Factors & $(\%)$ & $35(81.4)$ & 0.412 \\
Hypertension & $14(82.4)$ & $9(20.9)$ & 0.824 \\
Diabetes Mellitus & $2(11.8)$ & $6(14.0)$ & 0.495 \\
Smoking & $2(11.8)$ & $14(32.6)$ & 0.346 \\
Hyperlipidaemia & $4(23.5)$ & $10(23.3)$ & 0.042 \\
Cardiac diseases & $6(35.3)$ & $7(16.3)$ & 0.899 \\
Alcohol & $7(41.2)$ & $7(16.3)$ & 0.370 \\
Previous stroke & $3(17.6)$ & $2(4.7)$ & 0.370 \\
Contraceptive use & $0(0.0)$ & $2(4.7)$ & 0.145 \\
Sickle cell disease & $0(0.0)$ & $5(11.6)$ & 0.023 \\
Family history of stroke & $0(0.0)$ & $0(0.0)$ & \\
Undetermined & $2(11.8)$ & & \\
\hline
\end{tabular}

Table 3:

\begin{tabular}{|c|c|c|c|}
\hline Variables & Ischaemic Stroke & Control & P-value \\
\hline WBC $\times 10^{9} / \mathrm{I}$ & $6.1 \pm 1.3$ & $4.9 \pm 1.2$ & $<0.001$ \\
\hline Neutrophil x 10/l & $3.3 \pm 1.1$ & $2.3 \pm 0.5$ & $<0.001$ \\
\hline Lymphocytes x $10^{9} / 1$ & $2.1 \pm 0.6$ & $2.1 \pm 1.2$ & 0.809 \\
\hline Monocytes x 109/1 & $0.6 \pm 0.3$ & $0.4 \pm 0.2$ & 0.008 \\
\hline Haemoglobin (g/dl) & $11.9 \pm 1.7$ & $14.1 \pm 0.8$ & $<0.001$ \\
\hline $\operatorname{MCV}(\mathbf{f l})$ & $87.9 \pm 4.8$ & $82.4 \pm 6.8$ & $<0.001$ \\
\hline МCH (pg) & $27.3 \pm 1.8$ & $27.2 \pm 2.7$ & 0.852 \\
\hline MCHC (g/dl) & $31.2 \pm 1.4$ & $33.0 \pm 1.7$ & $<0.001$ \\
\hline Platelet $\times 10^{9} / 1$ & $235 \pm 94$ & $235 \pm 89$ & 0.997 \\
\hline
\end{tabular}

Table 4: Correlation between APLA and

\begin{tabular}{|c|c|c|c|c|c|c|c|c|c|c|}
\hline & & WBC & GRA & LYMPH & OTHERS & HB & MCV & МСH & МСНС & PLT \\
\hline \multirow[t]{2}{*}{ APLA } & $\mathrm{R}$ & -0.045 & -0.108 & 0.057 & -0.099 & 0.038 & -0.081 & 0.173 & 0.193 & 0.004 \\
\hline & $P$ value & 0.732 & 0.412 & 0.666 & 0.449 & 0.772 & 0.536 & 0.186 & 0.139 & 0.973 \\
\hline
\end{tabular}

$\mathrm{r}$ = Spearman correlation coefficient, WBC: white cell count; GRA: granulocytes; LYMPH: lymphocytes; OTHERS: monocytes, eosinophils and basophils; HB: haemoglobin; PCV: packed cell volume; MCV; mean corpuscular volume; $\mathrm{MCH}$ : mean corpuscular haemoglobin; $\mathrm{MCHC;}$ mean corpuscular haemoglobin concentration; PLT: platelets 